\title{
Revisions of Australian ground-hunting spiders: III. Tuxoctenus gen. nov. (Araneomorphae: Zoridae)
}

\author{
Robert J. Raven \\ Queensland Museum, PO Box 3300, South Brisbane, Queensland 4101, Australia. \\ Email: Robert.Raven@qm.qld.gov.au
}

\begin{abstract}
A new genus of the family Zoridae, Tuxoctinls, is described from mainland Australia and includes T. gloverae sp. nov (type species), T. limmer sp. nov. and T. modomaldae sp. nov. A key to Australian zorid genera is provided.
\end{abstract}

\section{INTRODUCTION}

In the Australian predominantly xeric landscape, a number of spider families have diverged strongly. Best documented amongst these are the gnaphosoids (Platnick 2000, 2002; Platnick and Baehr 2006), the Zodariidae (e.g., Baehr 2004) and perhaps also the Lycosidae (e.g., Framenau it al. 2006). Amongst the lesser known ground-hunting spiders are the Zoridae, best field-diagnosed perhaps by their high speed and common diurnal preference for open sunny spaces. The entire family is presently being revised in Australia but one very distinctive new monotypic genus stands out. The opportunity to honour Carolus Linnaeus, another "very distinctive genus", presented itself and the pair seemed to fit.

\section{MATERIALS AND METHODS}

Material was examined using a Stereodissecting microscope (Zeiss Stemi SV6 or SV11) with a camera lucida. Scanned material were either critical-point or air dried from alcoholpreserved material and then sputter-coated with gold before examination in an Hitachi S-530 scanning electron microscope sometimes using a Robinson (T) Backscatter detector. Epigynes were photographed in alcohol and then either cleared in lactic acid and drawn. The left palp is described with the ventral face uppermost. Eye group size is measured through the centre of the group and compared to the carapace width on that line. Abbreviations and methods are standard for the Araneae and explained in Raven and Stumkat (2003). Colour is described in $70 \%$ ethanol and, where known, that in life. Spine values suffixed with " $w$ " signify that the spines were weak and attenuate. Leg measurements are given for femur, patella, tibia, metatarsus (except pedipalp), tarsus, and total length.

The specimens examined for this study are lodged in the following institutions: $A M N H$,
American Museum of Natural History, New York; AMS, Australian Museum, Sydney; CAS, California Academy of Science, San Francisco; MACN, Museo Argentino de Ciencias Naturales, Buenos Aires; MV, Melbourne Museum, Victoria; QM, Queensland Museum, Brisbane; WAM, Western Australian Museum, Perth.

\section{SYSTEMATICS}

\section{Family Zoridae F.O.P.-Cambridge 1893}

\section{Diagnosis}

Eight eyes in two recurved rows or three rows with back eyes in two rows as in many Lycosidae; from front, ALE at same level as AME or higher but clearly closer to AME than ALE; grateshaped tapetum (at least in Argoctemus) which degrades quickly on spider's death. Two claws with claw tufts present or absent with no hairs around claws (Hestimodema); leg scopula weak or absent. Retrocoxal hymen distinct on coxa 1 . Males without fracture on pedal tibia, without cymbial scopula or unsclerotized region on tibial apophysis. C-shaped tegulum; basal to basolateral embolic origin for half circumference of bulb; single distal median apophysis; conductor short, membranous. Females with spigots only apical on PMS. Strong and often long paired spines (2-7 pairs) on tibiae $1, I I, 2-3$ pairs on metatarsi 1, II. (Taken from Raven and Stumkat 2003, with modifications)

\section{Included genera (Australian region)}

Argoctenus L. Koch 1878, Odomasta Simon 1909, Hestimudema Simon 1909, Elassoctemus Simon 1909, Thasuraen L. Koch 1878, Simonus Ritsema 1881, Tuxoctemes gen. nov.

\section{Key to known Australian zorid genera}

1. Ail eyes of similar size........................................ Eyes of posterior row much larger than those of 
anterior row or at least ALE..... .4

2. Eyes small and widely spaced 3

Eyes larger and more or less touching

Hestimodema

3. Abdomen elongate with long stripes dorsally .. Thasyrhea

Abdomen short, ovoid to pentagonal, dorsal stripes absent.

Odomasta

4. Only 2-4 pairs of weak spines on tibiae I and II

5-7 pairs of spines on tibiae I and II. .5

5. Pair of longitudinal ridges of white hair on dark carapace and abdomen (Figure 1a); PLE very large Tuxoctenus

Carapace and abdomen not as above; PME similar in size to PLE

Argoctenus

6. Carapace and abdomen with longitudinal stripes, paired spines on tibiae I and II, lateroventral and very strong.....

Simonus

Carapace and abdomen without stripes; tibial spines weak in males and females

Elassoctenus

\section{Tuxoctenus gen. nov.}

\section{Type species}

Tuxoctenus gloverae sp. nov.

\section{Diagnosis}

Tuxoctenus differs from all other Australian zorids except Argoctenus in that the posterior ocular quadrangle is about 1.7 times wider behind than in front and 1.2 times wider behind than in front (Figure 1a). Specimens can be easily recognised in life by the pair of longitudinal ridges of white hairs on an otherwise dark carapace and abdomen (Figure 1a).

\section{Description}

Longitudinal bands of white hairs on cephalothorax and abdomen. Eight eyes in 3 rows; front row recurved, ALE larger; PLE much the largest of all; line through midpoint of back row is ca. 0.6 of head-width at that point. Serrula absent. Chelicerae with 2 retromarginal and 3 promarginal teeth; small boss. Trochanters all with short deep notches. Coxae lack enlarged setae. Males with distinct spine on distal point of patellae III, IV. Scopula absent in males, weak to absent in females. True claw tufts. Weak paired spines ventrally on tibiae and metatarsi I, II. Male palp: deep cymbium with apical crest of bristles. Spinnerets short; PLS and ALS separated; apical article of PLS short. Epigynal plugs present. Male. Cymbium short, spoon-like with subtle

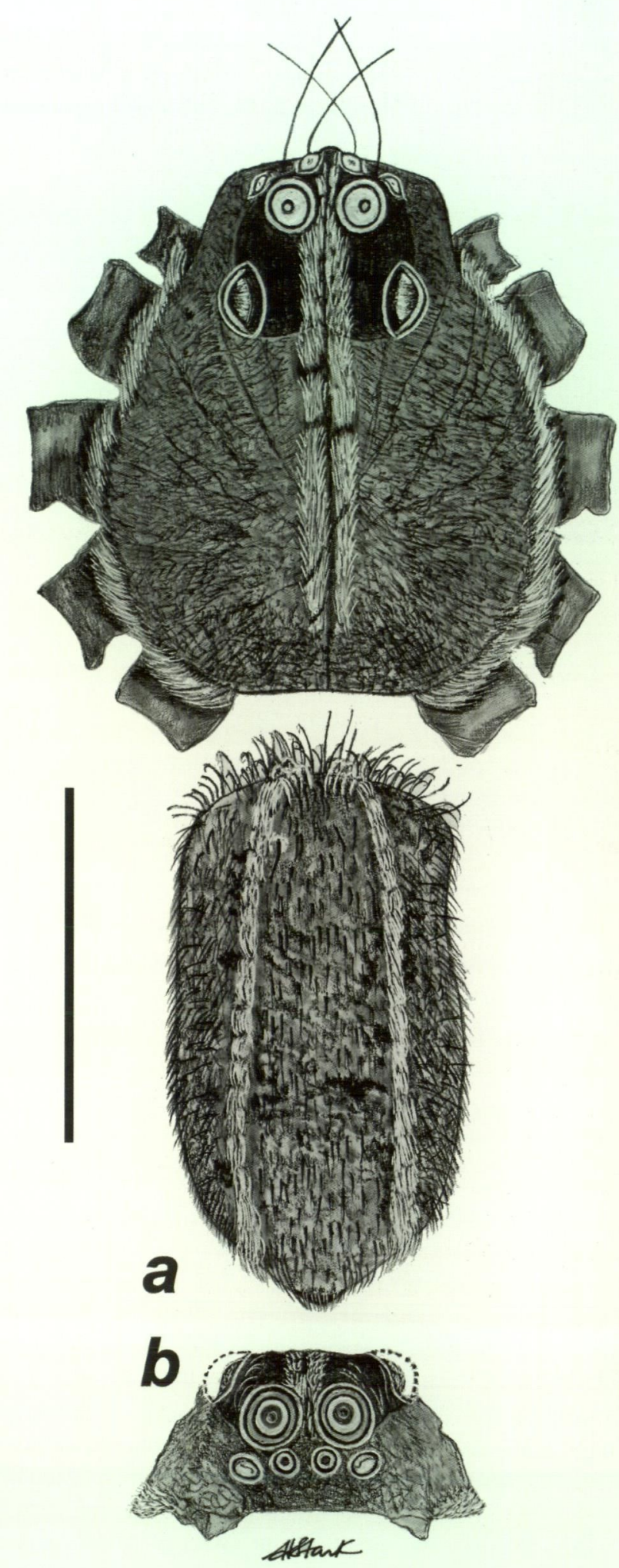

Figure 1 Tuxoctenus gloverae sp. nov., male: a, carapace and abdomen, dorsal view; b, "face", frontal view with PLE smaller through foreshortening. Scale line $=1 \mathrm{~mm}$.

basal groove along retrolateral margin, and short rounded apex with band of long recurved thick setae (Figure 4) extending from ventral subdistally over the tip and subdistally dorsally. RTA small, somewhat flattened, rhomboid, distally truncate 

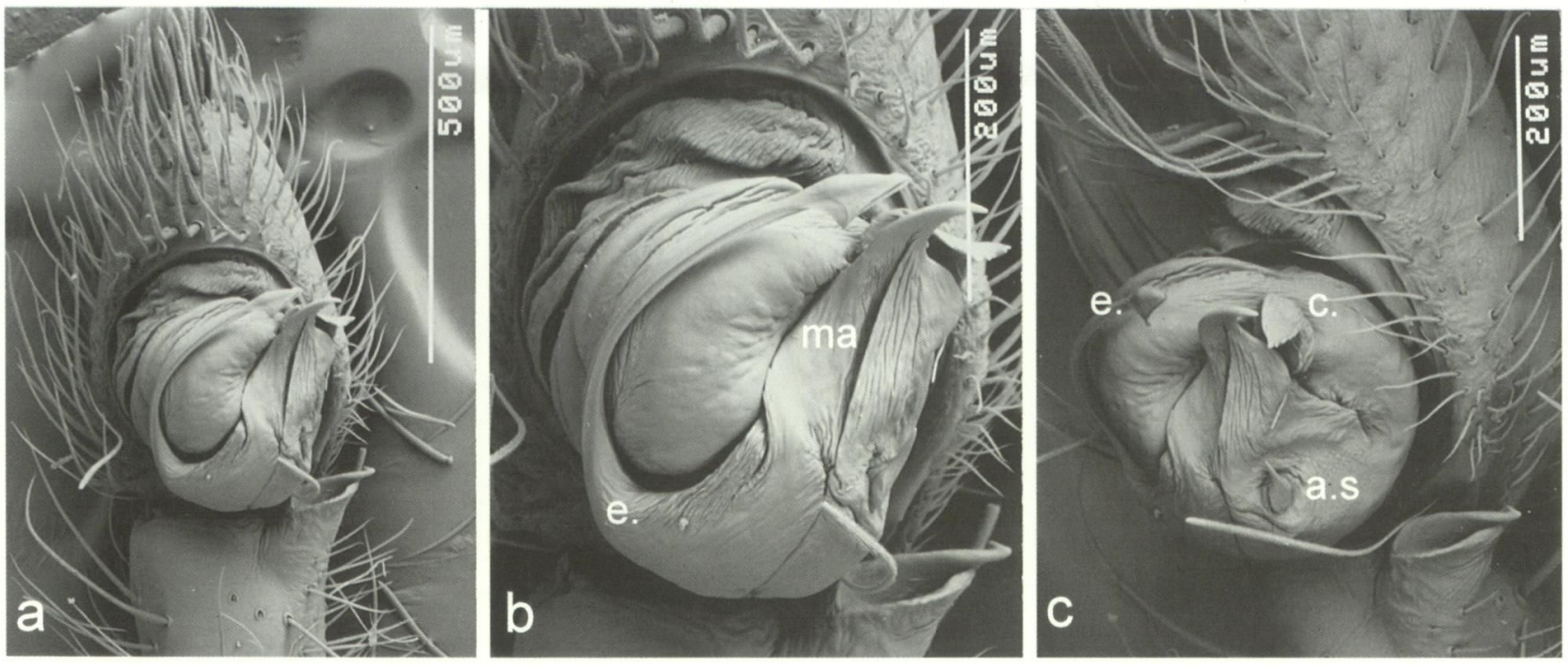

Figure 2 Tuxoctenus gloverae sp. nov., male tibia, cymbium and palpal bulb: a, b, ventral view; c, apical view. Abbreviations: a.s, accessory sclerite; c., conductor; e., embolus; ma, median apophysis.

and grooved, with small apical hook. Conductor distinct, opposed by long, flat and apically hooked median apophysis. Small to moderately large accessory sclerite in unsclerotised window at base of median apophysis (T. linnaei, T. gloverae or absent (T. mcdonaldae). Tegulum basal, large, divided into two similar (T. mcdonaldae, T. gloverae) or dissimilar lobes ( $T$. linnaei) but long anterior extension of basal embolus which is fused to median apophysis; retrolateral half of tegulum with triangular plate apically fused to prolateral edge of median apophysis. Embolus almost straight, originates prolaterobasally, narrow, fluted for length with flared pointed apex (Figure 2b). Palpal tibia cylindrical, patella without apophyses.

\section{Species included}

Tuxoctenus gloverae sp. nov., T. linnaei sp. nov., T. mcdonaldae sp. nov.

\section{Distribution and habitat}

Specimens of Tuxoctenus have been found across most of north-eastern, eastern southern and western Australia, and is apparently absent from Tasmania. Spiders have been found in moist litter pockets in open forest but usually not in rainforest. The highest densities were noted in heath on North Stradbroke Island, Queensland.

\section{Remarks}

The most distinct differences between males of species of Tuxoctenus are the presence, shape and size of a small accessory sclerite on the lower face of the median apophysis of the palp. Differences in the bulb and RTA are quite subtle. Slight differences are evident also in the spination of metatarsi I and II and the extent of the bushy white hairs on the carapace. Equally, in females, the biggest sexual difference is in the shape of the median septum of the epigyne which varies from broad strap-like (T. linnaei) or medially rounded (T. mcdonaldae) and diamond-shaped (T. gloverae).

The enlarged setae over the apical cymbium (Figure 4) are quite different in structure to spines and are not considered homologous.

In life, males of $T$. gloverae have a black carapace and abdomen; however, that pigment seems to quickly leach from specimens after preservation leaving a reddish brown to dark brown cuticle.

The absence of the serrula is unusual in zorids: Argoctenus, Simonus, Hestimodema and Thasyrhea all have a short straight serrula which is otherwise absent only in Odomasta.

\section{Etymology}

From the tuxedo-like appearance of the living spider and the ctenid-like eye pattern.

Tuxoctenus gloverae sp. nov.

Figures 1a, b, 2 a-c, 3 a, b, 4a-c, 9

Material examined

Holotype

Australia: Queensland: ô, Mt Coot-tha, Brisbane, $27^{\circ} 28^{\prime} \mathrm{S}, 152^{\circ} 57^{\prime} \mathrm{E}$, pitfall, 4 November 1996-4 July 1997, R.J. Raven (QM S32998).

\section{Allotype}

Australia: Queensland: + , Beerwah Forestry Reserve, $26^{\circ} 51^{\prime} \mathrm{S}, 152^{\circ} 57^{\prime} \mathrm{E}$, heathland, 24 April 1991, M. Glover (QM S32434). 


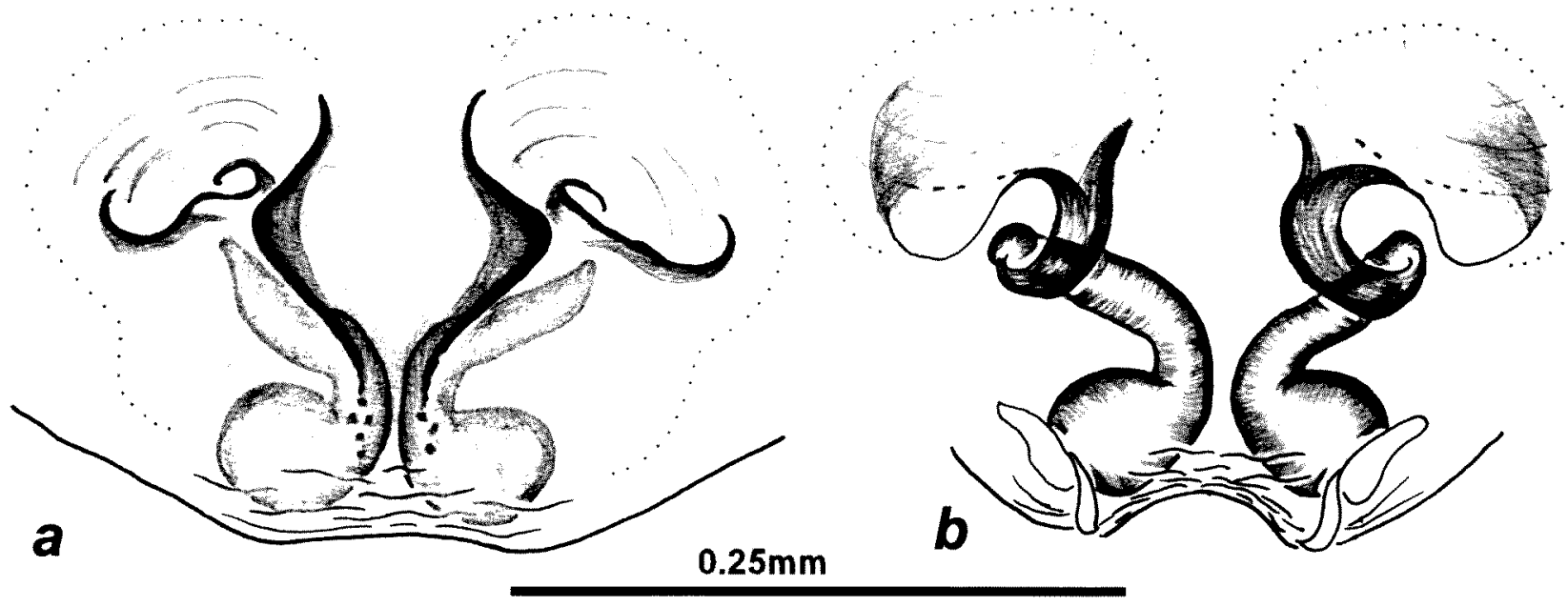

Figure 3 Tuxoctenus gloverae sp. nov, female epigyne of QM S56371: a, external; b, internal.

\section{Paratypes}

Australia: Queensland: 2 \&, Beerwah Forestry Reserve, 26 $51^{\prime} \mathrm{S}, 152^{\circ} 57^{\prime} \mathrm{E}$, heathland, 29 August 1990, pitfall, M. Glover (QM S39013, S39036); 2 o, same data except 16 May 1990 (QM S39027, QM S39008); 1 o, same data except 6 June 1990 (QM S39024); 1 d, same data except 18 July 1990 (QM S39032); 1 t, same data except 19 September 1990 (QM S39025); 4 8, same data except 10 October 1990 (QM S39022, S39038, S39016, S39029); 1 o., same data except 31 October 1990 (QM S39019); 20 , same data except 21 November 1990 (QM S39035, S39009); 4 ठ same data except 12 December 1990 (QM S39034, S39006, S39007, S39017); 1 o, same data except 2 January 1991 (QM S39021); 1 \%, same data except 27 February 1991 (QM S39011); 12 \&, same data except 24 April 1991 (QM S39012, S39023, S39015, S39026, S39010, S39020, S39030, S39041); 2 \%, same data except 26 June 1991 (QM S39028); 1 bे, same data except 25 August 1991 (QM S39033); 3 o, 1 \%, same data (QM S39018, S39014, S39037, S39031); 4 ơ, Coal Creek, Kholo, 273- $34^{\prime} \mathrm{S}, 152^{\circ} 44^{\prime} \mathrm{E}$, open forest, pitfall, 11 November 1998-13 January 1999, G. Monteith, D. Cook, G. Thompson (QM S59719); 3 d, same data except 13 January-16 May 1999, G. Monteith (QM S49998, S52262); 1 o, Enoggera Reservoir, site $1,27^{\circ} 26^{\prime} \mathrm{S}, 152^{\circ} 55^{\prime} \mathrm{E}$, open forest, pitfall, 21 December 1999-27 January 2000, G. Monteith, J. Holt (QM S55842); 1 o, 1 \&, same data except site 1 27 January-15 March 2000 (QM S55833); 1 3, same data except flight intercept trap, 27 January-15 March 2000 (QM S55836); 1 o, same data except site 2 pitfall, 21 December 1999 -27 January 2000 (QM S55848); 1 \%, same data except 16 October-21 December 1999, G. Monteith (QM S55832); 16 oै, 8 q, North Stradbroke Island, Enterprise Mine site, Blackbutt sites 2-3, 27 $33-34^{\prime} \mathrm{S}, 1^{\circ} 3^{\circ} 26-7^{\prime} \mathrm{E}$, hand collection, 9 January 2002, Queensland Museum
Party (QM S55481, S55448); 29 ठ, 2 \&, same data except pitfall, 8-22 January 2002 (QM S55584); 6 0, 4 \%, North Stradbroke Island, Enterprise Mine site, Mallee sites $1-3,27^{\circ} 34-5^{\prime} \mathrm{S}, 153^{\circ} 26^{\prime} \mathrm{E}$, hand collection, 8-22 January 2002, Queensland Museum Party (QM S55437); 15 o, same data except pitfall (QM S55583); 1 \%, same data except hand collection (QM S56302); 1 o, same data except hand collection (QM S55963); 4 \%, 1 \&, same data except (QM S55451); 1 oे, 1 q, same data except (QM S55428); 1 d, North Stradbroke Island, Enterprise Mine site, Scribbly Gum sites $1-3,27^{\circ} 35-6^{\prime} \mathrm{S}, 153^{\circ} 26-7^{\prime} \mathrm{E}$, hand collection, 9-10 January 2002, D.J. Cook (QM S55593); 2 \&, 2 q, same data except 10 January 2002 (QM S56371); $230,3 q$, same data except pitfall, 8-22 January 2002 (QM S55585); 16 o, 17 \%, same data except 10 January 2002, Queensland Museum Party (QM S55500, AMNH, CAS, MACN, WAM); 1 ${ }^{\star}$, Ewan Maddock Dam, $26^{\circ} 47^{\prime} \mathrm{S}, 152^{\circ} 58^{\prime} \mathrm{E}$, open forest, pitfall, 22 August 1993-22 March 1994, M. Glover (QM S39040); 1 ס, same data except 27 October 1992-30 January 1993 (QM S39039); 7 , Mt Coot-tha, Brisbane, $27^{\circ} 28^{\prime} \mathrm{S}, 152^{\circ} 57^{\prime} \mathrm{E}$, pitfall, 4 November 1996-4 July 1997, R.J. Raven (QM S31372); $14 \delta$. Mulgowie, Laidley Valley, $27^{\circ} 44^{\prime} \mathrm{S}$, $152^{\circ} 22^{\prime} \mathrm{E}, 25$ March 1981, M.D. Grant (QM S42738); 1 d., 1 q, same data (QM S42737); 1 , Pine Mt Environmental Park, $27^{\circ} 32^{\prime} \mathrm{S}, 152^{\circ} 41^{\prime} \mathrm{E}$, open forest, pitfall, 13 January-16 May 1999, G. Monteith (QM S49991). New South Wales: 9 8, $0.5 \mathrm{~km}$ from Wheatly Creek Road on Camp Creek Road, $28^{\circ} 47^{\prime} \mathrm{S}, 1^{\circ} 2^{\circ} 19^{\prime} \mathrm{E}, 550 \mathrm{~m}, 4$ February-9 April 1999, National Park Survey, M. Gray, G. Cassis (AM KS36267).

\section{Diagnosis}

Males differ from those of $T$. modonaldae by the presence of a small but distinct accessory sclerite 

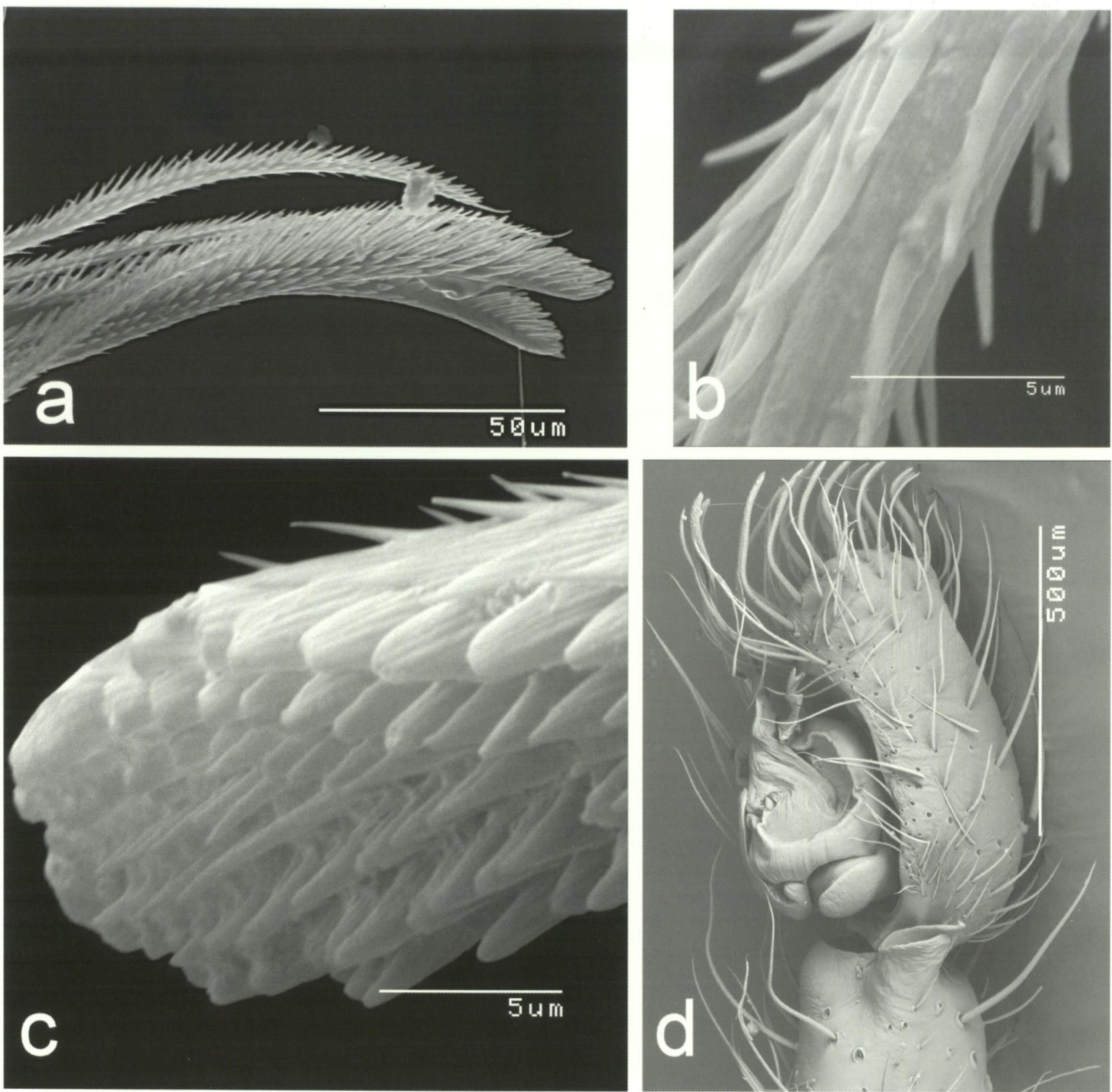

Figure 4 Tuxoctenus gloverae sp. nov., male palp: a-c, enlarged setae on cymbial tip, apical length (a), shaft (b), and flattened tip (c); d, tibia, cymbium and palpal bulb, retrolateral view.

in the tegular window (Figure 2c) and only one pair of spines ventrally on metatarsi I, II; females differ from other species by the diamond-shaped median septum (Figure 3a).

\section{Description}

\section{Holotype male (QM S32998)}

Measurements: Carapace 1.56 long, 1.34 wide. Abdomen 1.65 long, 0.83 wide. Leg I: 1.64, 0.68, $1.44,1.36,1.00,6.12$. Leg II: $1.56,0.56,1.36,1.24$, 1.16, 5.68. Leg III: $1.52,0.60,1.20,1.28,1.16,5.44$. Leg IV: $2.12,0.64,1.84,2.16,0.47$, 7.92. Palp: 0.76, $0.24,0.28,-, 1.76$

Colour: Carapace uniformly dark reddish brown with dense bush of long, erect bright white hairs in marginal bands and 2 close medial bands. Chelicerae lighter without bands. Legs grey black without bands or mottling. Abdomen dorsally black (from dense short black hair), anteriorly with long recurved bristles and two divergent bright white bands from dorsal corners posteriorly; ventrally with paler central medial quadrangle and 4 pairs of even paler spots. Sternum dark grey; maxillae and labium grey; labium with pallid anterior lip and inner faces of maxillae pallid. Coxae greenish black with pallid basal 'fingers' laterally and medially.

Carapace: Pear-shaped with short anterior constriction; eye group extends back beyond 
constriction; caput indistinct; lateral cephalic profile flat between fovea and clypeus. Pile of short black hairs on carapace, lateral band of white hairs very distinct, longer hairs reaching from anterior constriction back to posterior cephalic corner. Medial bands composed of shorter white hairs from AME just onto posterior slope. Long recurved black bristle between $\mathrm{AME}$ and below it on clypeus edge; long bristle in front of each ALE and a pair on each side lateral of that. Fovea short, enclosed by white bands. Eyes. Eight in 3 rows; all but ALE circular. From above, front row clearly recurved, eyes of similar size (about 1/3-1/2 of PME) but ALE larger; from front, slightly but clearly recurved; front row width little under that of MOQ back width; AME not on mound and much closer to each other than to ALE. Back eyes in 2 widely separated rows; PME "look" up, and to front and side, PME much closer to each other than to PLE; PLE look side and slightly back. From front, PLE larger and set lower than AME, clypeus ca. 2-3 diameter AME. ALE oval, look down and out.

Chelicerae: Small, vertical; boss very small, pallid narrow. 3 bands of only $10-15$ bristles along chelicerae. Fang short; dentition 3P, 2R.

Maxillae: Short rectanguloid, apically truncate thickest medially with pallid anterior and inner ledges. Serrula absent. Labium vertical, short with lateral corners to shield behind incrassate anterior.

Sternum: Rounded, shield-like, rounded corners at intercoxae, flat with only ca. 60 long erect hairs totally; precoxal sclerites absent.

Legs: Coxae with distinct anterior projections basally, less distinct posteriorly. Small retrocoxal hymen centrally on posterior face of coxa I. Black feathery hairs and long black bristles on femora, patellae and tibiae. Legs with iridescent dark green sheen. Long erect bristle on distal corner of patellae and tibiae I, II. Scopula mostly absent but scopuliform hairs prolaterodistally on tarsi. Tarsi elongate, slightly curved down. All trochanters with short deep notches. 2-3 hairs on retrodistal ventral coxae, III longer. Shield or comb of bristles on ventral trochantera strongest posteriorly on III, IV. Trichobothria. Tarsi with 2 rows each of 4-6, longest distally; metatarsi with $6-8$, one very long, bent, distally; tibiae with 2 rows for full length. Spines. Weak ventrally on tibiae and metatarsi I, II; strongest on III, IV. Leg I: fe pv1p1d3r2; pa 0 ; ti v2.2.2w; me v2 basal. Leg II: fe p2d3r3; pa 0 ; ti v2.2.2w; me v2 basal. Leg III: fe p2d3r3; pa d1 apical; ti p2d2r2v2.2.2; me p2.1r2.1v2.1. Leg IV: fe p2d3r2; pa d1 apical; ti p2d2r2v2.2.2; me p2.2d1r2.2v2. Palp: fe p1d1.1.1; pa pld2 (one apical); ti p2d1r2; cymbium no spines. Claws long, curved, with 3-5 teeth. Tufts dense, distinct.
Spinnerets: ALS short, barrel-like, separated. PLS short, thin, ca. 1/3 diameter of ALS; PMS same size as PLS but slightly thinner.

Palp: Tibia incrassate almost from base; RTA on retroventral corner, sclerotised ridge forms small hook above large unsclerotised area basally with truncate ledge. Cymbium almost rectangular, deep; apical portion rounded, truncate but not tapered; a crest of 2 lines of long thick recurved black bristles from ventral cymbial edge medially onto dorsum, bristles shortest dorsally; margins narrow rounded with retrobasal ridge. Tegulum extensive; apical cap with unsclerotised islands retrolaterally and basally. Median apophysis diagonal with small curved hook apically; basally with lachrymoidal sclerite in ovoid unsclerotised tegular window. Embolus with wide but partially unsclerotised base prolaterally arching across to small translucent cushion retrolaterally.

\section{Allotype female (QM S32434)}

Measurements: Carapace 1.46 long, 1.23 wide. Abdomen 1.96 long, 1.31 wide. Leg I: 1.18, 0.66 1.02, 0.81, 0.44, 4.01. Leg II: 1.14, 0.50, 0.96, 0.79, 0.62 4.01. Leg III: $1.14,0.48,0.81,0.87,0.73,3.82$. Leg IV: $1.56,0.54, .129,1.43,0.73,5.55$. Palp: 0.46, 0.27, 0.31, - $0.52,1.56$.

Colour: Carapace brown with narrow white margin and median bands; abdomen dorsally grey centrally lighter with two distinct white bands widening to back, venter grey with 4 long pale lines. Legs light brown, no bands; eyes on black tubercles except ALE set off.

Legs: No scopula. Dense claw tufts; tarsi bowed with pallid apex. Distal-most metatarsi trichobothrium 1.5 times longer than penultimate trichobothrium; all trichobothria on tibiae long for full length. Palpal tarsus tapering, conical. Spines: leg I: fe pv1p1d2; pa 0 ; ti v2.2w; me v2w. II fe p1d3; pa 0; ti v2.2w v2w. III: fe p2d3r1; pa 0 ; ti p2d2r2v2.2; me p2r2v2.2. IV: fe p1d3r1; pa apical; ti p2d2r3v1.2; me p1.1.1.2r2v1. Palp: fe d1.2; pa d2 + 1 apical; ti p3d2; ta p5d1r2 plus v2 subapical. Claws. Long, curved, slender, set well above tufts with 3-5 teeth. Palpal claw long curved, clear of hair with 3 teeth.

Spinnerets (from QM S55836): PLS longer than ALS which are subequal to PMS. Apical segment of PLS clearly truncate.

Epigyne: Anteriorly two broadly U-shaped ridges mark entrance of copulatory pores, medially appears to have broad convergent septum but actually the strongly sclerotised basal portion of spermathecae which are elongate sigmoida with dorsal thumb-like lobe and rectanguloid fertilisation ducts basally. 


\section{Distribution and habitat}

This species is found throughout litter in eucalypt forest and open woodland throughout south-east Queensland; it has not been recorded from rainforest.

\section{Etymology}

The species epithet is a patronym in honour of Mrs Maureen Glover, Honorary Associate of the Queensland Museum, whose enthusiasm for arachnology resulted in a large collection of $T$. gloverae from heathlands just north of Brisbane.

\section{Tuxoctenus linnaei sp. nov.}

Figures $5 \mathrm{a}-\mathrm{c}, 6 \mathrm{a}, \mathrm{b}, 8,9$

\section{Material examined}

\section{Holotype}

Australia: South Australia: ô, between Nundroo and Nullarbor Stations, 28 February 1978, B.Y. Main (WAM 99/2388).

\section{Allotype}

Australia: South Australia: $q$, same data as holotype (WAM 99/2389).

\section{Other material examined}

Australia: Western Australia: $1+$, Burma Road, edge of Nature Reserve, $29^{\circ} 00^{\prime} \mathrm{S}, 115^{\circ} 04^{\prime} \mathrm{E}$, Banksia litter, 11 April 1992, M. Harvey (WAM T74020); 2 §, Stirling Range National Park, White Gum Flat, $34^{\circ} 22^{\prime} S, 117^{\circ} 48^{\prime} \mathrm{E}, 3$ February-6 March 1979, M.R. Gray (AM KS15930).

\section{Diagnosis}

Males of T. linnaei differ from those of T. gloverae by the large triangular sclerite in the tegular window; females differ from both T. gloverae and T. modonaldae in the almost parallel-sided median septum on the epigyne.

\section{Description}

\section{Holotype male (WAM 99/2388)}

As for T. gloverae, except as follows:

Measurements: Carapace 1.22 long, 1.14 wide. Abdomen 1.57 long, 1.06 wide. Leg I: 1.37, 0.51, 1.21, 1.02, 0.66, 4.77. Leg II: 1.33, 0.66, 1.05, 1.05, 0.74, 4.84. Leg III: 1.17, 0.59, 1.05, 0.66, 0.63, 4.10. Leg IV: $1.64,0.55,1.56,1.84,0.78,6.37$. Palp: 0.66, 0.20, 0.31, -, 0.55, 1.72 .

Colour: Carapace uniformly dark reddish brown with fine black radial lines in striae and bushy
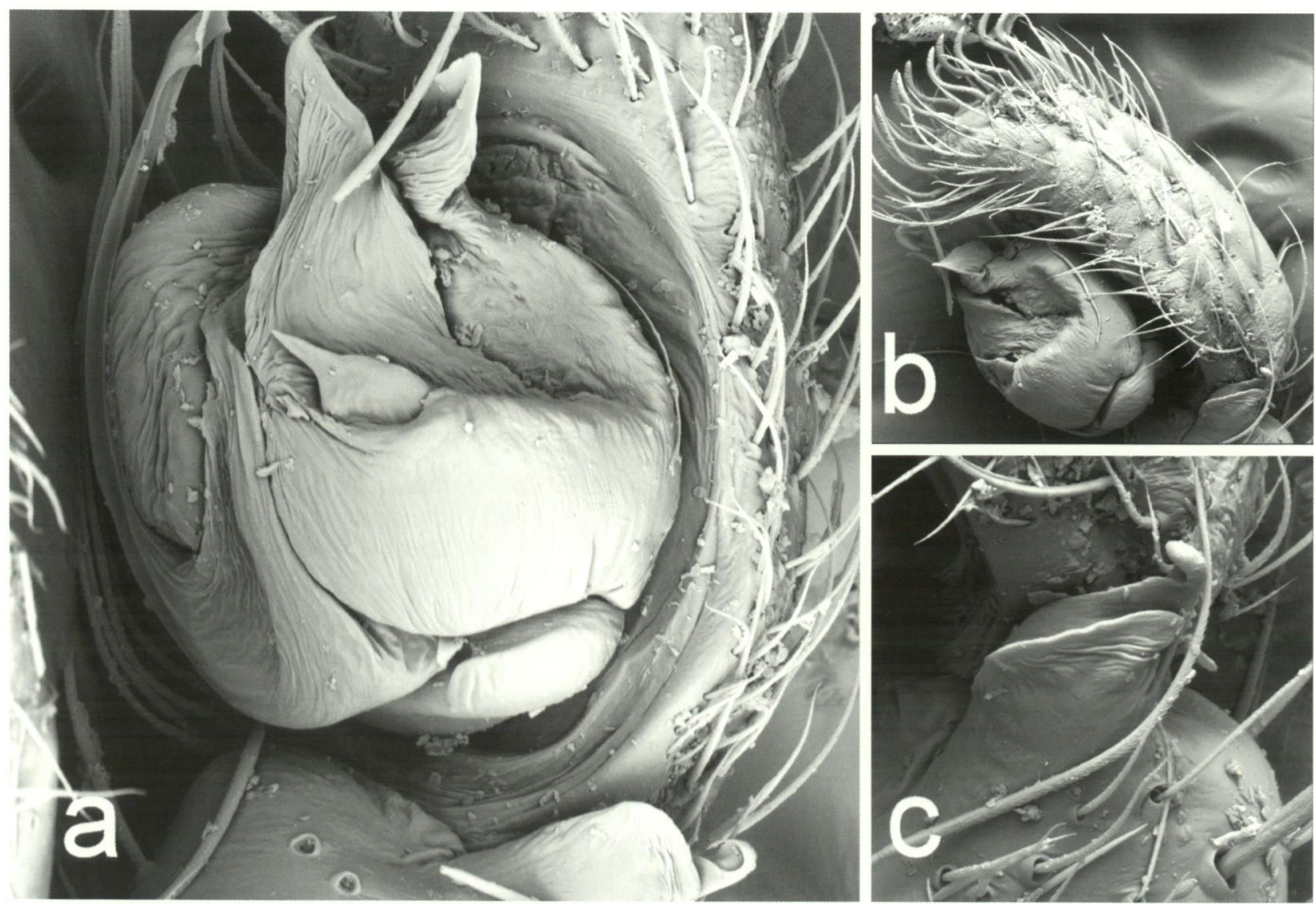

Figure 5 Tuxoctenus linnaei sp. nov., AMKS 15930, male tibia, cymbium and palpal bulb: a, ventral view; b, retrolateral view; c, RTA, retrolateral view. 


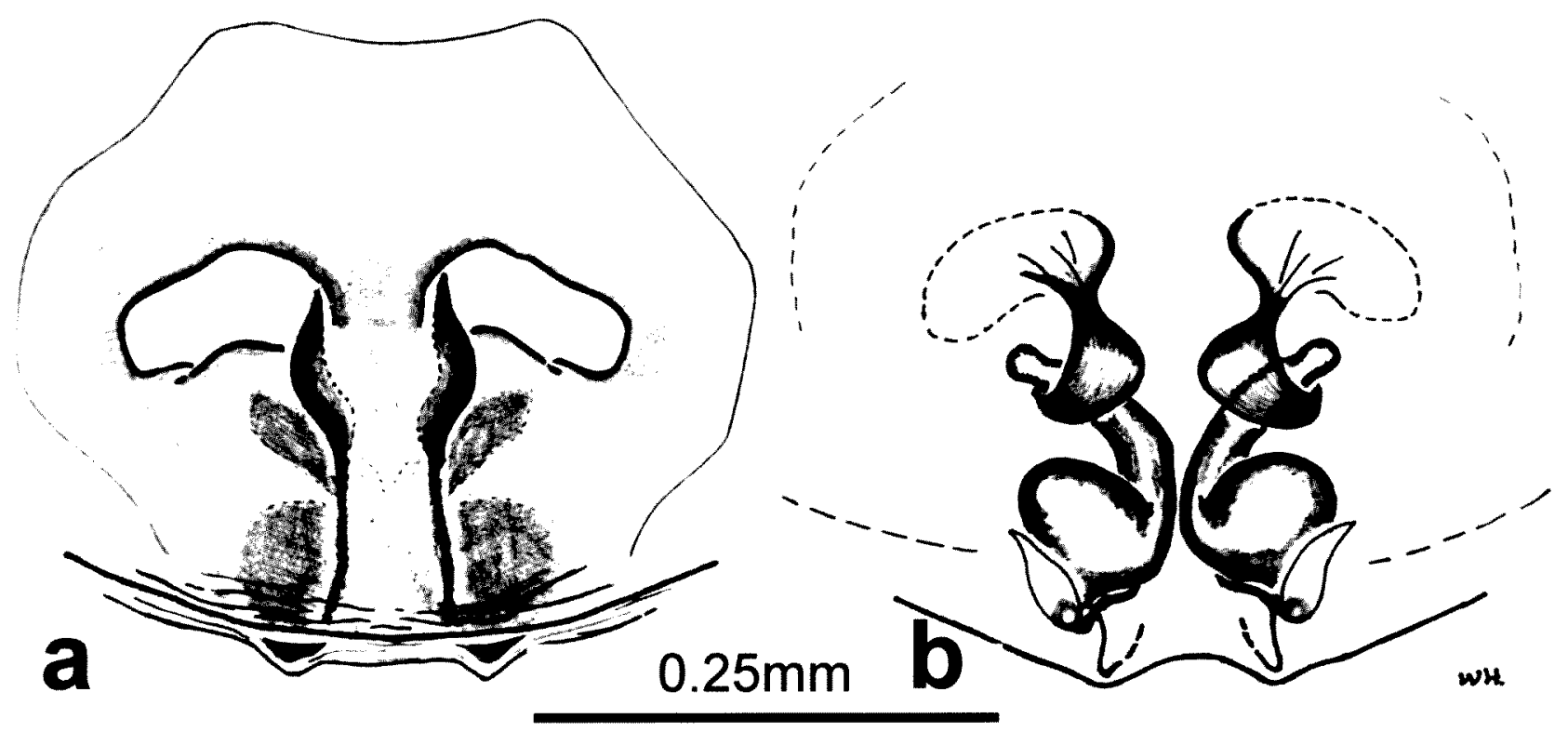

Figure 6 Tuxoctenus linnaei sp. nov, female epigyne of allotype: a, external; b, internal.

bands of long bright white hairs along margins and small patch between PME. Chelicerae and legs brown, not banded. Abdomen dorsally brown, anteriorly with two white lines slightly divergent posteriorly to back corner; ventrally pallid centrally with brown flanks. Sternum, maxillae and labium pallid.

Carapace: Light pile of fine black hairs on carapace. Pair of long porrect bristles below ALE and off caput corners; $2-3$ long bristles lateral of ALE. Chelicerae anteriorly with $4-5$ very long downcurved bristles at midlength. Eyes: Oval ALE similar in diameter to round AME. From front, front row straight.

Legs: Black feathery hairs not evident. Trochantera with short distinct notches. Weak comb of bristles on ventral trochantera III, IV. Spines: Leg I: fe p1d3r1; pa 0; ti v2.2.2w; me v2.2. Leg II: fe p1d3r2; rest as leg I. Leg III: fe p2d3r1; pa d1; ti p2d2r2v2.2.2w; me p2.1r2.1v2.1. Leg IV: fe p2d3r1; pa d1, r1; ti p2d2r2v2.2.2; me p2.2r1.2.1v2.

Palp: fe p1d1.1.2; pa pld1; ti p3d2r1; cymbium 0 but with two parallel rows, each of 6 thick recurved bristles. Bulb: as $T$. gloverae but tegular window filled with triangular sclerite. RTA short, recurved, scythe-like.

\section{Allotype female (WAM 99/2389)}

Measurements: Carapace 1.84 long, 1.24 wide. Abdomen 2.54 long, 1.39 wide.

As in male. Most legs missing.

Epigyne. Large wide medial septum, almost parallel-sided, with pair of recurved ridges set anteriorly marking copulatory openings. Internally, very similar to $T$. gloverae.

\section{Distribution}

Tuxoctenus linnaei is known only from the Nullarbor Plain, South Australia and Stirling Ranges and Burma Road Nature Reserve, southwestern Western Australia.

\section{Etymology}

This species is named in honour of Carolus Linnaeus, the founder of modern taxonomy.

\section{Tuxoctenus modonaldae sp. nov.}

Figures $7 a-c, 8 a, b, 9$

\section{Material examined}

Holotype

Australia: Queensland: o, Osbourne Mine site, SSE. of Mt Isa (site 2a), $22^{\circ} 07^{\prime} \mathrm{S}, 140^{\circ} 34^{\prime} \mathrm{E}$, open forest, pitfall, 19 April-2 July 1996, R. Raven, A. Nicholson (QM S79287).

\section{Allotype}

Australia: Queensland: $q$, same data as holotype (QM S79288).

\section{Paratype}

Australia: Queensland: $1 \delta$, same data as holotype (QM S31231).

\section{Other material examined}

Australia: Queensland: Brigalow Research Station, Theodore, $24^{\circ} 48^{\prime} \mathrm{S}, 149^{\circ} 45^{\prime} \mathrm{E}, 16$ December 2000-28 March 2001, D. Cook, G. Monteith: 1 3. flight intercept trap site 3, pitfall site 3 (QM S56452); 1 o, same data (QM S55847); 1 d, flight 

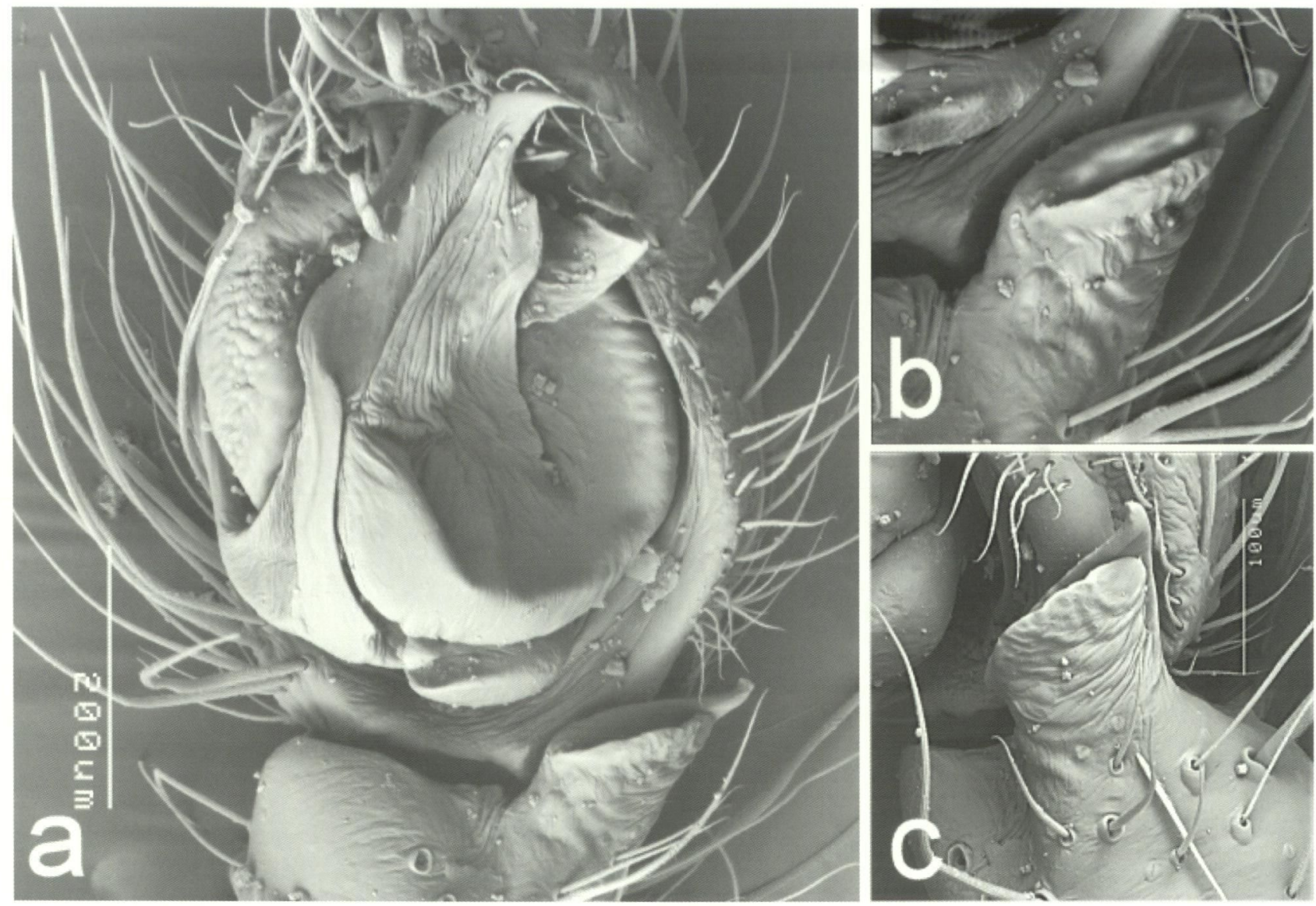

Figure 7 Tuxoctenus mcdonaldae sp. nov., male tibia, cymbium and palpal bulb: a, ventral view; b, c, RTA, slightly apica view (b) and retrolateral view (c); all at same scale.

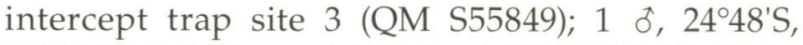
$149^{\circ} 47^{\prime} \mathrm{E}$, brigalow, pitfall site 4 (QM S55843); flight intercept trap site 5) $20^{\circ}$, pitfall site 5, $24^{\circ} 48^{\prime} \mathrm{S}$, $149^{\circ} 45^{\prime} \mathrm{E}$ (QM S55844); 1 to (QM S56388). 1 के, Bushley Station (DW12), 2331'S, 150 ${ }^{\circ} 14^{\prime} \mathrm{E}$, open forest, pitfall, 3 September 1991, D. Wallace, R. Raven (QM S39044); 1 d, Mazeppa National Park, north end, $22^{\circ} 13^{\prime} \mathrm{S}, 147^{\circ} 15^{\prime} \mathrm{E}$, brigalow, pitfall, 18 December 2000-26 March 2001, D. Cook, G. Monteith (QM S56445); 2 \$, 1 \&, same data except 18 December 2000-26 March 2001, D. Cook, G. Monteith (QM S59774); 1 d, Mazeppa National Park, south end, $2216^{\prime} \mathrm{S}, 147^{\circ} 16^{\prime} \mathrm{E}$, brigalow, flight intercept trap, 18 December 2000-26 March 2001, D. Cook, G. Monteith (QM S59786); 1 § 5 km S. of Moranbah, $22^{\circ} 02^{\prime} \mathrm{S}, 148^{\circ} 02^{\prime} \mathrm{E}$, pitfall, 20 December 1997-26 April 1998, G. Monteith (QM S57030); 2 o, $7 \mathrm{~km}$ NNE. of Mt Bluffkin, on highway, $22^{\circ} 35^{\prime} \mathrm{S}, 149^{\circ} 14^{\prime} \mathrm{E}$, brigalow, flight intercept trap, 19 December 2000-25 March 2001, D. Cook, G. Monteith (QM S55841); 3 oे, Tooloombah Creek

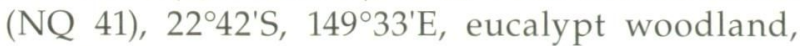
pitfall, 29 July-24 November 1992, R. Raven, P. Lawless, E. Lawless, M. Shaw (QM S24509); 1 + , same data except 10 November 1991-29 July 1992 (QM S24084); 7 6, Windemere Station, Glenmorgan, $27^{\circ} 17-25^{\prime} \mathrm{S}, 149^{\circ} 41-44^{\prime} \mathrm{E}$, December
1990-October 1991, R. Raven, B.J. Smyth (QM S39042); 1 o, 1 \%, same data except (QM S39043); 1 ô, 1 i, same data (QM S51904); 1 ô, same data (QM S31489). Western Australia: 2 के, Nerren Nerren Station (NE4), 2700'21.6"S, 114'32'29.2"E, wet pitfalls, 11 January-11 May 1995, P. West et al. (WAM T88440) [2 males T88439, perhaps same data, no other label]; 2 , Nerren Nerren Station

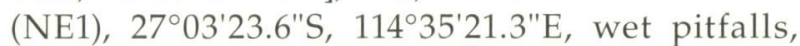
11 January-11 May 1995, P. West et al. (WAM T88441); 1 d, Cape Cuvier, Quobba Station (CU5), $24^{\circ} 11^{\prime} 34.0^{\prime \prime S}, 113^{\circ} 27^{\prime} 17.4^{\prime \prime}$, wet pitfalls, 15 January-29 May 1995, A. Sampey et al. (WAM T88438).

\section{Diagnosis}

Males of T. mcdonaldae differ from those of $T$. gloverae by the absence of a sclerite in the tegular window and only two pairs of spines ventrally on metatarsi I, II; in females, the median septum of the epigyne is similar to that of $T$. gloverae but the lateral procurved ridges are set much more posteriorly.

\section{Description}

\section{Holotype male QM S79287}

As for $T$. gloverae, except as follows. 


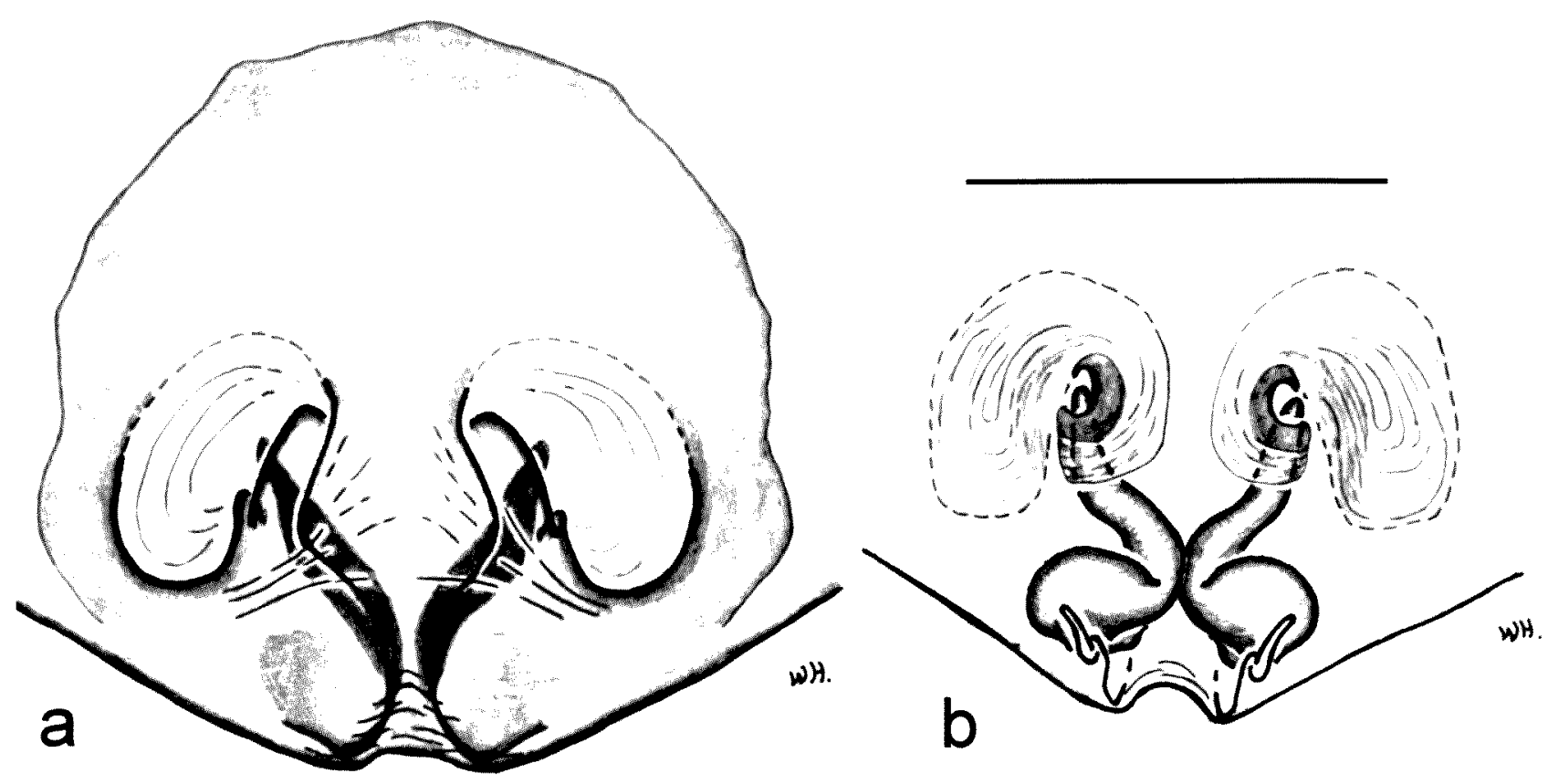

Figure 8 Tuxoctenus mcdonaldae sp. nov., female epigyne of allotype: a, external; b, internal.

Measurements: Carapace 1.63 long, 1.41 wide. Abdomen 1.41 long, 0.78 wide. Leg I: 1.53, 0.72, 1.47, 1.38, 0.72, 5.81. Leg II: 1.41, 0.63, 1.34, 1.19, 0.63, 5.19. Leg III: $1.47,0.50,1.22,1.03,0.69,4.91$. Leg IV: $2.03,0.69,1.81,1.94,0.78,7.25$. Palp: 0.66, 0.34, 0.38, $-, 0.66,2.03$

Colour: Carapace uniformly light brown with light bush of long white hairs in marginal bands and one medial band; chelicerae and legs light brown, without bands. Abdomen dorsally faded black (from dense short black hair) laterally with pallid median stripe, anteriorly with long recurved bristles and two divergent white bands from dorsal corners posteriorly; ventrally with two lines of paler spots laterally. Sternum, maxillae and labium pallid.

Legs: Comb of bristles ventrally on trochantera distinct on III, IV. Spines. I: fe pv1pld3r2; pa 0 ; ti v2.2.2w; me v2.2. II: fe p1d3r2; pa 0 ; ti v2.2.2w; me v2.2. III: fe p1d3r2; pa d1; ti p2d2d1r2v2.2.2; me p2.1r2.1v2.1. IV: fe p2d3r2; pa d1; ti p2d1r3v2.2.2; me p1.2d1.1r2.1v2.2.2. Palp: fe p1d1.1.2; pa p1d2; ti p2d1r1; cymbium 0 .

Palp: Inner edge of RTA sclerotised with small apical hook, outer face a translucent vane form deep hollow with inner edge. Cymbium rectanguloid in dorsal view; a crest of 2 lines of 9-19 long recurved black bristles in each line. Tegulum extensive for distal half above hooked median apophysis. Median apophysis diagonal basally with trianguloid unsclerotised window without sclerite; embolus narrow tubular for length.

\section{Allotype female (QM S79288)}

Measurements: Carapace 1.75 long, 1.34 wide. Abdomen 2.03 long, 1.53 wide. Leg I: 1.38, 0.68, 1.03, 0.97, 0.63, 4.69. Leg II: $1.34,0.63,1.03,0.94,0.53$, 4.47. Leg III: $1.41,0.66,0.94,0.94,0.38,4.31$. Leg IV: both missing. Palp: 0.66, 0.31, 0.34, - 0.56, 1.88 .

Colour: As male but ventral abdomen pallid, possibly damaged. Carapace pilosity like male of T. linnaei.

Legs: Scopuliform hairs on distal half of metatarsi I, II, and weak but evident for length of tarsi I, II. Spines. I: fe pld3; pa 0; ti v2.2; me v2w. II: as for I. III: fe p2d2r1; pa 0; ti p2d2r2v2.2; me p2r2v2. IV, missing. Palp: fe p1d1.2w; pa d2.1; ti p3d2; ta $22 \mathrm{r} 2+2$ subapical.

Epigyne: Median septum of the epigyne is similar to that of $T$. gloverae but lateral procurved ridges set much more posteriorly.

\section{Distribution and habitat}

This species is known from north-western Queensland (Cammoweal) through xeric areas near Claremont, Mazeppa National Park and Windemere Station and north western Western Australia at Nerren Nerren station and Cape Cuvier. The localities include diverse kinds of xeric habitats from grassland with scattered eucalypts to brigalow.

\section{Etymology}

This species is named in honour of Mrs Sue McDonald, Warren Vale Station, Normanton, north-western Queensland, through whose 


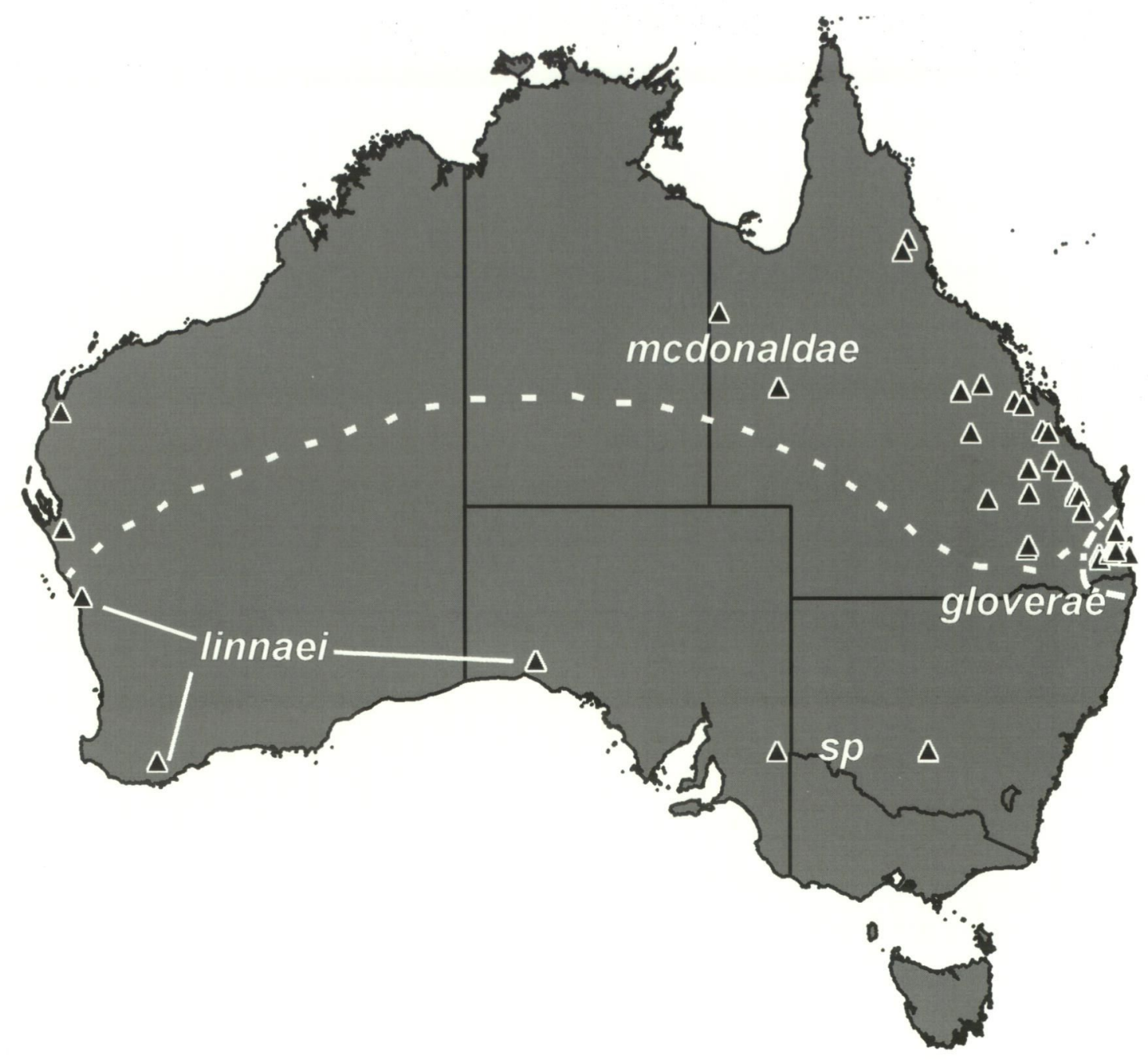

Figure 9 Tuxoctenus records.

seemingly endless patience for the authors and enthusiasm for spiders the QM made exciting discoveries in arachnids in the Gulf region.

\section{ACKNOWLEDGEMENTS}

Drs Barbara Baehr and V. Davies, Queensland Museum, provided support and counterpoint through the early phases. Mrs Helen Stark skilfully illustrated Figure 1. Mrs Wendy Hebron, Queensland Museum, very ably helped with registration and sorting of material and the wonderful epigyne illustrations (Figures 2, 6, 8). Finally, grateful thanks to Dr Mark Harvey, friend and colleague, for the encouragement and especially for the last minute support to complete this work.

\section{REFERENCES}

Baehr, B.C. (2004). The systematics of a new endemic Australian genus of ant spiders Masasteron (Araneae: Zodariidae). Invertebrate Systematics 18: 661-691.

Framenau, V.W., Gotch, T.B. and Austin, A.D. (2006). The wolf spiders of artesian springs in arid South
Australia, with a revalidation of Tetralycosa (Araneae, Lycosidae). Journal of Arachnology 34: 1-36.

Platnick, N.I. (2000). A relimitation and revision of the Australasian ground spider family Lamponidae (Araneae: Gnaphosoidea). Bulletin of the American Museum of Natural History 245: 1-330.

Platnick, N.I. (2002). A revision of the Australasian ground spiders of the families Ammoxenidae, Cithaeronidae, Gallieniellidae, and Trochanteriidae (Araneae: Gnaphosoidea). Bulletin of the American Museum of Natural History 271: 1-243.

Platnick, N.I. and Baehr, B. (2006). A revision of the Australasian ground spiders of the family Prodidomidae (Araneae, Gnaphosoidea). Bulletin of the American Museum of Natural History 298: 1-287.

Raven, R.J. (1994). Mygalomorph spiders of the Barychelidae in Australia and the western Pacific. Memoirs of the Queensland Museum 35: 291-706.

Raven, R.J. and Stumkat, K. (2003). Problem solving in the spider families Miturgidae, Ctenidae and Psechridae (Araneae) in Australia and New Zealand. Journal of Arachnology 31: 105-121.

Manuscript received 20 June 2008; accepted 2 October 2008. 